\title{
SEMIPARAMETRIC IDENTIFICATION IN DURATION MODELS
}

Andrew Chesher

THE INSTITUTE FOR FISCAL STUDIES DEPARTMENT OF ECONOMICS, UCL 


\title{
Semiparametric identification in duration models
}

\author{
Andrew Chesher* \\ Centre for Microdata Methods and Practice \\ Institute for Fiscal Studies and University College London
}

November 13th 2002

\begin{abstract}
This paper explores the identifiability of ratios of derivatives of the index function in a model of a duration process in which the impact of covariates on the hazard function passes through a single index. The model allows duration and the index to appear in a nonseparable form in the hazard function and includes a latent heterogeneity term which acts multiplicatively on the hazard function. The model allows covariates to be endogenous, that is to be correlated with the heterogeneity term. Quantile invariance, local order and local rank conditions are shown to be sufficient to permit identification of ratios of derivatives of the index function. The framework constructed in this paper is suitable for the analysis of identification in panel duration models with heterogeneity.
\end{abstract}

\section{INTRODUCTION}

This paper considers the identification of features of processes generating duration data. A model is proposed in which the hazard function at duration $t$ conditional on the value, $x$, of observed time constant covariates and the value, $u$, of an unobserved latent variable, $\lambda_{T \mid X U}(t \mid x, u)$, is a nonseparable function of $t$ and a scalar index $\theta(x)$ with $u$ having a proportionate effect, as follows.

$$
\lambda_{T \mid X U}(t \mid x, u)=j(t, \theta(x)) / u
$$

The model does not embody the familiar "proportionate hazard restriction" on the impact of covariates on the hazard function but it does impose this restriction on the impact of the term representing uncontrolled heterogeneity. The aim of this paper is to determine weak conditions under which interesting features of the conditional hazard function can be identified. In practice one may impose additional restrictions in order to facilitate estimation. The results of this paper allow one to see which restrictions imposed have fundamental identifying power, and which do not. This is important knowledge as Roehrig (1988) persuasively argues.

The paper focusses on the identification of the value of a ratio of derivatives of the index function with respect to variables $x_{i}$ (numerator) and $x_{j}$ (denominator) when these variables are exogenous (but others may not be) and, more interestingly, when a variable $x_{i}$ is endogenous and $x_{j}$ is exogenous ${ }^{1}$. For the purpose of this paper a variable $x_{\rho}$ is exogenous if the conditional distribution of the latent heterogeneity variable, $U$, given $x$ is invariant with respect to changes in $x_{\rho}$. When this condition does not hold $x_{\rho}$ is endogenous.

The endogenous covariate could be a duration in a previous spell. So, the analysis can be interpreted as providing conditions under which features of panel duration structures

\footnotetext{
${ }^{*}$ I am grateful to Simon Sokbae Lee for helpful comments.

${ }^{1}$ Extension to the case with more than one endogenous variable in $x$ is straightforward using the methods of this paper and is not considered here.
} 
are identified. But this is not the only application because the endogenous variable could be an associated outcome of the duration process, for example, in a labour supply setting, the wage paid in a job held prior to an unemployment spell, or the reservation wage ${ }^{2}$.

This is an analysis of semiparametric identification because the model proposed is, apart from the index restriction on the impact of covariates and the proportionality restriction on the heterogeneity term, nonparametrically specified. The analysis is conducted in the context of a system of simultaneous structural equations with values of outcomes (the duration of interest and the other endogenous variable) arising as the solution to the structural equations at observed values of covariates and unobserved values of latent random variables. This is the construction proposed by Hurwicz (1950).

This leads to a triangular nonseparable (in the latent variables) simultaneous equation system of the sort studied in Chesher (2001a, 2001b, 2002a, 2002b). A distinctive feature of duration models with heterogeneity is that there are more sources of stochastic variation than observed outcomes which was disallowed in these papers.

A crucial implication of the proportionate heterogeneity restriction is that two of the sources of stochastic variation combine, free of the covariates to produce a system with the same number of observable outcomes as there are sources of stochastic variation, a system to which the methods of those papers do apply.

As noted in Chesher (2001a) analysis of identification in nonseparable models is naturally approached by considering the relationship between the structural features of interest (in this case ratios of derivatives of index functions) and functionals of conditional quantile functions derived from the joint distribution of observable outcomes given covariates.

Under certain monotonicity restrictions on the structural functions, restrictions on the conditional quantiles of the distributions of latent variates given covariates imply restrictions on conditional quantile functions involving observable outcomes. These conditional quantile restrictions on the latent random variables, together with order and rank type conditions on the impact of covariates on the structural functions are embodied in the identifying model proposed in this paper. Manski (1988), in the context of binary response models, argues elegantly and persuasively for consideration of conditional quantile restrictions as identifying restrictions, and conditional quantile identifying restrictions are used in problems close to that considered here by Chaudhuri, Doksum and Samarov (1997), Matzkin (1999) and Kahn (2001).

The model considered here requires the impact of $X$ to pass through a single ${ }^{3}$ index, $\theta(X)$, a restriction imposed in many other papers including, Han (1987), Powell, Stock and Stoker (1989), Newey and Stoker (1993), Sherman (1993) and Chaudhuri, Doksum and Samarov (1997) and Kahn (2001). This restriction is imposed in this paper because it implies that interesting structural features (ratios of index derivatives) are free of stochastic variation. In the model studied here, in which there are two, potentially confounded, sources of variation (duration and heterogeneity related) there can be ambiguity in the interpretation of structural features which do not have this property.

Chesher and Lancaster (1984) considered parametric duration models in which hazard functions depend upon endogenous variables and Lancaster (1985) examined identification in such a model using an approximate linearisation which cast the problem into the classical linear simultaneous equations form in which the role of order (exclusion) and rank conditions in achieving identification is clear. Local versions of these conditions feature in the identifying restrictions set out in this paper. The attack on the identification problem taken in this paper, via conditional quantile functions, removes the need for the linear approximation and the restriction to specific functional forms (e.g. for hazard func-

\footnotetext{
${ }^{2}$ See Chesher and Lancaster (1983) and Lancaster (1985) for examples of analysis of data of this sort.

${ }^{3}$ In fact the analysis could proceed using multiple indexes, $\theta_{1}\left(X_{1}\right), \theta_{2}\left(X_{2}\right)$, etc., as long as attention is restricted to identification of index derivative ratios involving pairs of covariates that appear in just one of the indexes.
} 
tions) employed in Lancaster (1985). It suggests an alternative attack on the estimation problem considered by Lancaster (1985) based on conditional quantile regression function estimation in place of the two stage least squares procedure he employed which was based on an approximate linearisation of the structural equations.

Koenker and Geling (2001) appraise the utility of a conditional quantile regression attack on the analysis of durations and their dependence on conditioning variables. Regarded in the context of this paper, Koenker and Geling's analysis is a "reduced form" analysis in the sense that heterogeneity is not modelled explicitly, the focus being directly on the conditional distribution of a duration given covariates and the information about this that is imparted by estimated quantile regression functions. In that context there is no role for an "endogenous" variable affecting duration because there is no structural model involving heterogeneity with which such an "endogenous variable" could be correlated.

The main result of the paper is contained in Section 4. A ratio of derivatives of the index function at some point of interest is shown to be identified by the proposed model and its value is delivered by a functional of conditional quantile functions involving only observable random variables. This suggests that conditional quantile estimation methods (Koenker and Bassett (1978)) could have more potential in the analysis of duration data than is perhaps generally appreciated.

The plan of the paper is as follows. Section 2 introduces the essential duration related elements of the model, specifying the admissible form of the hazard function in Section 2.1. Section 2.2 recalls the definition of identifiability of a structural feature due to Hurwicz (1950), and then in Section 2.3 the problem is set in Hurwicz structural equation form. Section 3 introduces additional restrictions producing a model which identifies a ratio of index function derivatives when covariates are exogenous. Section 4 introduces a final set of restrictions producing a model that identifies ratios of index function derivatives when a covariate is endogenous. Estimation issues and the impact of censoring are discussed at the end of Section 4 and Section 5 concludes.

Restrictions are introduced as they are needed in the exposition. The complete set of restrictions is set out for reference in Annexes 1 and 2, respectively covering the exogenous and the endogenous case.

\section{HAZARD MODELS WITH PROPORTIONATE HETEROGENEITY}

2.1. Hazard function. Let the hazard function for absolutely continuous scalar random variable $T \in(0, \infty)$ given $k$-element $X=x$ and scalar $U=u>0$ be

$$
\lambda_{T \mid X U}(t \mid x, u)=\lambda_{T \mid \theta(X) U}(t \mid \theta(x), u)=j(t, \theta(x)) / u
$$

where $\theta(x)$ is a scalar function of $x$. The integrated hazard function is $J(t, \theta(x)) / u$ where

$$
J(t, \theta(x))=\int_{0}^{t} j(s, \theta(x)) d s, \quad j(s, \theta(x))=\nabla_{t} J(t, \theta(x)) .
$$

This paper develops conditions additional to (1) under which the relative sensitivity of the index $\theta(x)$ to variations in two elements of $x$ is identifiable. This relative sensitivity is the ratio of derivatives

$$
R_{i j}(x)=\frac{\nabla_{X_{i}} \theta(x)}{\nabla_{X_{j}} \theta(x)}
$$

and throughout consideration is given only to cases in which, at the value $x$ considered, the denominator derivative is nonzero. The model embodies the restriction that the index function is differentiable with respect to $X_{i}$ and $X_{j}$ at $X=x$.

With the dependence of the hazard function on $t$ and $\theta(x)$ nonparametrically specified as in (1) one of the derivatives $\nabla_{X_{j}} \theta(x) \neq 0$ can always be normalised to equal 1 with 
suitable modification of the function $j(t, \theta(x))$, and with that done, $R_{i j}(x)$ measures the sensitivity of the normalised index to variations in $X_{i}$ at $X=x$.

2.2. Identification. The ratio of derivatives $R_{i j}$ is identified by a model (dependence on $x$ is suppressed in the notation for the present) when it takes the value $R_{i j}^{*}$ if, for any structure, $\mathcal{S}$, admitted by the model in which $R_{i j}=R_{i j}^{*}$, the value of $R_{i j}$ is $R_{i j}^{*}$ in all admissible structures observationally equivalent to $\mathcal{S}$ (Hurwicz (1950). Structures observationally equivalent to $\mathcal{S}$ generate the same conditional distribution of $T$ given $X$, $F_{T \mid X}$, as is generated by $\mathcal{S}$.

The Lemma in Chesher (2002b) states that $R_{i j}$ is identified when its value is $R_{i j}^{*}$ if there exists a functional $\mathcal{G}\left(F_{T \mid X}\right)$ with the property that all admissible structures with $R_{i j}=R_{i j}^{*}$ generate conditional distributions for $T$ given $X$ such that $\mathcal{G}\left(F_{T \mid X}\right)=R_{i j}^{*}$.

The strategy adopted in this paper is to determine weak restrictions on structures, that is a model, sufficient for the existence of a functional $\mathcal{G}(\cdot)$ with this property. Estimation of the value of an identifiable $R_{i j}$ follows directly using the analogue estimator $\mathcal{G}\left(\hat{F}_{T \mid X}\right)$.

2.3. Hurwicz structural form. It is convenient to cast the duration problem studied in this paper in the structural equation - latent disturbance form set out in Hurwicz (1950) and employed in Lancaster (1985). In this construction $M$ observable outcomes (e.g. $T$ and when they may arise, other endogenous variables) are regarded as being determined by $M$ equations delivering values of observable outcomes given values of $N$ latent unobservable random variables and values of covariates. A structure comprises a particular system of equations and a particular conditional distribution for the latent variables given the covariates.

To cast the problem of this paper in this form, first note that the conditional distribution function of $T$ given $X=x$ and $U=u$ is

$$
F_{T \mid X U}(t \mid x, u)=1-\exp (-J(t, \theta(x)) / u)
$$

and the associated conditional $p$-quantile function is

$$
Q_{T \mid X U}(p \mid x, u)=J^{-1}(w(p, u), \theta(x))
$$

where

$$
w(p, u)=-u \log (1-p)
$$

and $J^{-1}(a, b)$ is the unique solution to

$$
a=J\left(J^{-1}(a, b), b\right) .
$$

Note that $w(p, u)$ is a strictly increasing function of $u$ and $p$ and that since $T$ is continuously distributed $J(a, b)$ is strictly increasing in $a$ for any value of $b$ and so there is, for any $b$, always a unique solution to (2).

Let $V$ be uniformly distributed on $(0,1)$. Then the random variable $T$ can be written in Hurwicz structural form as

$$
T=J^{-1}(W(V, U), \theta(X))
$$

where the random variable $W(V, U)$ is defined as

$$
W(V, U) \equiv-U \log (1-V) .
$$

Given any particular value $x$ of $X$, and $u$ of $U$, and with $V$ uniformly distributed on $(0,1)$, the equation (3) delivers a random variable, $T$, whose conditional distribution function given $U=u$ and $X=x$ is

$$
F_{T \mid X U}(t \mid x, u)=1-\exp (-J(t, \theta(x)) / u) .
$$


Further, given any particular distribution of $U$ given $X, F_{U \mid X}$, and a value $x$ of $X$ the equation (3) delivers a random variable, $T$, with conditional distribution function given $X=x$, marginal with respect to $U$, as follows.

$$
F_{T \mid X}(t \mid x)=1-\int \exp (-J(t, \theta(x)) / u) d F_{U \mid X}(u \mid x) .
$$

The random variable $V$ is, by construction, independent of $(U, X)$.

The structural equation (3) generates $M=1$ outcome $(T)$ given $N=2$ sources of stochastic variation $(V$ and $U)$. The force of the proportionate heterogeneity restriction is that these two sources of stochastic variation combine, free of $X$, to a single source, $W(V, U)$. Lancaster (1985) noted this phenomenon in the parametric model he studied. The result is a consequence of the proportionate heterogeneity restriction and holds in wide class of duration models. That these two sources of stochastic variation do coalesce into a single source of variation via a functional relationship free of $X$ is crucial in the argument of Section 4.

\section{IDENTIFICATION UNDER LOCAL EXOGENEITY RESTRICTIONS}

For any $p \in(0,1)$ and some value $x$ at which identification of $R_{i j}(x)$ is sought, define

$$
\begin{aligned}
w(p, x) & \equiv Q_{W \mid X}(p, x) \\
t(p, x) & \equiv J^{-1}(w(p, x), \theta(x))
\end{aligned}
$$

where $Q_{W \mid X}(p, x)$ is the $p$-quantile of $W(V, U)$ given $X=x$.

Since $J^{-1}$ is strictly increasing in its first argument the equivariance property of quantiles ensures that the conditional $p$-quantile of $T$ given $X=x$ is

$$
Q_{T \mid X}(p, x)=t(p, x) .
$$

Thus the value of $t(p, x)$ is identified in the sense that for admissible structures ${ }^{4}$

$$
t(p, x)=t^{*} \Rightarrow Q_{T \mid X}(p, x)=t^{*}
$$

the functional referred to in Section 2.2 being $\mathcal{G}\left(F_{T \mid X}\right)=Q_{T \mid X}(p, x)$.

Consider an element, $X_{i}$, of $X$ which has continuous variation and let the first partial derivative of $t(p, x)$ with respect to $X_{i}$ be denoted by $t_{i}(p, x)$. Then

$$
t_{i}(p, x)=\nabla_{w} J^{-1}(w(p, x), \theta(x)) \nabla_{i} w(p, x)+\nabla_{\theta} J^{-1}(w(p, x), \theta(x)) \nabla_{i} \theta(x)
$$

where the operators $\nabla_{w}, \nabla_{\theta}$ and $\nabla_{i}$ indicate partial differentiation with respect to respectively $w(p, x), \theta(x)$ and $x_{i}{ }^{5}$

Consider two elements of $X, X_{i}$ and $X_{j}$ and the quantile invariance restrictions

$$
\nabla_{i} Q_{W \mid X}(p, x)=0 \quad \nabla_{j} Q_{W \mid X}(p, x)=0
$$

(which may only hold for the specified value $p$ and in a neighbourhood of the value of $x$ of interest), equivalently

$$
\nabla_{i} w(p, x)=0 \quad \nabla_{j} w(p, x)=0 .
$$

\footnotetext{
${ }^{4}$ The restrictions embodied in the identifying model under exogeneity are set out in Annex 1. Identification of the value of $t(p, x)$ requires only Condition 1 to hold. This result is essentially an application of a result in Matzkin (1999) which studies identification of nonparametrically specified functions of exogenous variables using conditional quantile restrictions.

${ }^{5}$ The requirement that $T$ has a continuous distribution ensures that $\nabla_{w} J^{-1}$ exists. The existence of other derivatives is embodied in the restrictions of the model set out in Annex 1.
} 
Under these restrictions, which can be thought of as local exogeneity restrictions,

$$
\begin{aligned}
t_{i}(p, x) & =\nabla_{\theta} J^{-1}(w(p, x), \theta(x)) \nabla_{i} \theta(x) \\
t_{j}(p, x) & =\nabla_{\theta} J^{-1}(w(p, x), \theta(x)) \nabla_{j} \theta(x)
\end{aligned}
$$

from which, if $\nabla_{\theta} J^{-1}(w(p, x), \theta(x)) \neq 0$, a further restriction now imposed,

$$
\frac{t_{i}(p, x)}{t_{j}(p, x)}=\frac{\nabla_{i} \theta(x)}{\nabla_{j} \theta(x)}=R_{i j}(x) .
$$

If $X_{i}$ and $X_{j}$ show continuous variation in a neighbourhood of the point $X=x$ and if the relevant quantile invariance conditions hold in a neighbourhood of the point $x$, then the value of $t(p, x)$ in a neighbourhood of $x$ is identified. From this it follows that at $x$ the derivatives $\nabla_{i} t(p, x)$ and $\nabla_{j} t(p, x)$ are identified, that is, in admissible structures:

$$
\begin{aligned}
& \nabla_{i} t(p, x)=t_{\nabla_{i}}^{*} \Rightarrow \nabla_{i} Q_{T \mid X}(p, x)=t_{\nabla_{i}}^{*} \\
& \nabla_{j} t(p, x)=t_{\nabla_{j}}^{*} \Rightarrow \nabla_{j} Q_{T \mid X}(p, x)=t_{\nabla j}^{*}
\end{aligned}
$$

It follows that the ratio of derivatives, $R_{i j}(x)$, of the index $\theta(x)$ is, under the restrictions imposed, and set out in Annex 1, identifiable, because in admissible structures,

$$
R_{i j}(x)=R^{*} \Rightarrow \frac{\nabla_{i} Q_{T \mid X}(p, x)}{\nabla_{j} Q_{T \mid X}(p, x)}=R^{*} .
$$

Note that there is no requirement that elements of $X$ other than $X_{i}$ and $X_{j}$ be locally exogenous, that is this identification result does not require restrictions of the sort

$$
\nabla_{l} w(p, x)=0 \quad l \notin\{i, j\} .
$$

An obvious estimator of the value of $R_{i j}(x)$ is the analogue estimator

$$
\hat{R}_{i j}(x)=\frac{\nabla_{i} \hat{Q}_{T \mid X}(p, x)}{\nabla_{j} \hat{Q}_{T \mid X}(p, x)}
$$

where $\hat{Q}_{T \mid X}(p, x)$ is an estimator of the $p$-quantile regression of $T$ given $x$.

Estimation could be done in the context of a semi-parametric single index model as currently stated, or, if that is too demanding of data then further, parametric restrictions could be imposed (and tested since they will be overidentifying restrictions). Estimations is discussed further in Section 4.4.

If the local exogeneity restrictions hold at more than one value of $p$ at the value of $x$ considered then $R_{i j}(x)$ is over identified and there is (a) scope for testing a subset of the identifying restrictions and (b) scope for improved efficiency in the estimation of the value of $R_{i j}(x)$, for example employing a minimum distance method to resolve non-concordant estimates got using different sets of just identifying restrictions. However, in practice it may be difficult to maintain the quantile invariance restrictions for one value of $p$, while allowing the restrictions to fail at other values - an issue taken up in Section 4.5.

\section{IDENTIFICATION WITH LOCAL ENDOGENEITY}

This Section considers the case in which one element of $X$ is endogenous in the sense that the conditional distribution of $U$ given $X=x$ depends on this element of $X$. This element of $X$ could be the duration of a previous spell, or some associated outcome. For example, if $T$ is the length of a spell of unemployment the endogenous element in $X$ could be the 
length of a previous spell of employment or unemployment, or the wage received in the job held prior to the unemployment spell.

Denote the endogenous element of $X$ by $Y$ and denote the remaining elements of $X$ by $Z$. The vector $X$ is now written as $(Y, Z)$, the index $\theta(X)$ is now written as $\theta(Y, Z)$ and the identifiability of the ratio of derivatives

$$
R_{Y j}(y, z)=\frac{\left.\nabla_{Y} \theta(Y, Z)\right|_{Y=y, Z=z}}{\left.\nabla_{Z_{j}} \theta(Y, Z)\right|_{Y=y, Z=z}}
$$

is considered where $y$ and $z$ are specified values of $Y$ and $Z$. These derivatives and others arising below are assumed to exist and to be finite and only cases in which

$$
\left.\nabla_{Z_{j}} \theta(Y, Z)\right|_{Y=y, Z=z} \neq 0
$$

are considered. ${ }^{6}$

The endogenous $Y$ is generated by the "reduced form" equation

$$
Y=h(Z, S)
$$

where $S$ is a latent unobservable random variable, not restricted to be distributed independently of $W(V, U)$, and $h(Z, S)$ is restricted to be strictly monotonic (normalised increasing) with respect to variations in $S$ in a neighbourhood of the value, $z$, of $Z$ at which identification of the ratio of derivatives $R_{Y j}(y, z)$ is sought.

The structural equations determining $T$ and $Y$ are therefore:

$$
\begin{aligned}
T & =J^{-1}(W, \theta(Y, Z)) \\
Y & =h(Z, S)
\end{aligned}
$$

where $W=W(V, U)$.

This is a triangular nonseparable pair of simultaneous equations determining values of $T$ and $Y$ given values of $W, S$ and $Z$, a special case ${ }^{7}$ of the model studied in Chesher (2001a, 2001b, 2002a, 2002b).

Consider $p_{Y} \in(0,1)$ and the $p_{Y}$-quantile of $S$ given $Z=z, Q_{S \mid Z}\left(p_{Y}, z\right)$. Denote this value of $S$ by $s\left(p_{Y}, z\right)$. Let

$$
y\left(p_{Y}, z\right) \equiv h\left(z, s\left(p_{Y}, z\right)\right) .
$$

This is the value of $Y$ at which identification of $R_{Y, i}(y, z)$ is sought. The remainder of this Section develops conditions under which the ratio of derivatives of the index function:

$$
\left.R_{Y j}(y, z)\right|_{y=y\left(p_{Y}, z\right)}=\frac{\left.\nabla_{Y} \theta(Y, Z)\right|_{Y=y\left(p_{Y}, z\right), Z=z}}{\left.\nabla_{Z_{j}} \theta(Y, Z)\right|_{Y=y\left(p_{Y}, z\right), Z=z}}
$$

is identified.

The first step is to show that under the conditions already stated the value of $y\left(p_{Y}, z\right)$ is identified.

4.1. Identification of $y\left(p_{Y}, z\right)$. The equivariance property of quantiles ensures that

$$
y\left(p_{Y}, z\right)=Q_{Y \mid Z}\left(p_{Y}, z\right) .
$$

and so, in admissible structures

$$
y\left(p_{Y}, z\right)=y^{*} \Rightarrow Q_{Y \mid Z}\left(p_{Y}, z\right)=y^{*}
$$

\footnotetext{
${ }^{6}$ The complete set of restrictions defining the identifying model under endogeneity are set out in Annex 2 .

${ }^{7}$ A special case because $S$ does not appear in its own right in the structural equation for $T$.
} 
and therefore the value of $y\left(p_{Y}, z\right)$ is identifiable under the restrictions so far imposed.

Consider $p_{T} \in(0,1)$, the value of $W$ :

$$
w\left(p_{T}, p_{Y}, z\right) \equiv Q_{W \mid S Z}\left(p_{T}, s\left(p_{Y}, z\right), z\right)
$$

and the value of $T$ :

$$
t\left(p_{T}, p_{Y}, z\right) \equiv J^{-1}\left(w\left(p_{T}, p_{Y}, z\right), \theta\left(y\left(p_{Y}, z\right), z\right)\right) .
$$

It is now shown that, under conditions to be stated, the ratio of derivatives of the index function (8) can be expressed as the following functional of derivatives of $t\left(p_{T}, p_{Y}, z\right)$ and $y\left(p_{Y}, z\right)$.

$$
\left.R_{Y j}(y, z)\right|_{y=y\left(p_{Y}, z\right)}=\frac{\nabla_{i} t\left(p_{T}, p_{Y}, z\right)}{\nabla_{j} t\left(p_{T}, p_{Y}, z\right) \nabla_{i} y\left(p_{Y}, z\right)-\nabla_{i} t\left(p_{T}, p_{Y}, z\right) \nabla_{j} y\left(p_{Y}, z\right)}
$$

Here the operators $\nabla_{i}$ and $\nabla_{j}$ indicate partial differentiation with respect to elements $z_{i}$ and $z_{j}$ of $z$.

The final step, taken in Section 4.3, is to show that under the stated conditions values of these derivatives are identified at the point of interest.

4.2. Re-expressing the ratio of index derivatives. The first partial derivative of $t\left(p_{T}, p_{Y}, z\right)$ with respect to an element of $z, z_{\rho}$ is as follows.

$$
\begin{aligned}
\nabla_{z_{\rho}} t\left(p_{T}, p_{Y}, z\right) \equiv & t_{\rho}\left(p_{T}, p_{Y}, z\right) \\
= & \nabla_{w} J^{-1}\left(w\left(p_{T}, p_{Y}, z\right), \theta\left(y\left(p_{Y}, z\right), z\right)\right) \nabla_{\rho} w\left(p_{T}, p_{Y}, z\right) \\
& +\nabla_{\theta} J^{-1}\left(w\left(p_{T}, p_{Y}, z\right), \theta\left(y\left(p_{Y}, z\right), z\right)\right) \nabla_{y} \theta\left(y\left(p_{Y}, z\right), z\right) \nabla_{\rho} y\left(p_{Y}, z\right) \\
& +\nabla_{\theta} J^{-1}\left(w\left(p_{T}, p_{Y}, z\right), \theta\left(y\left(p_{Y}, z\right), z\right)\right) \nabla_{\rho} \theta\left(y\left(p_{Y}, z\right), z\right)
\end{aligned}
$$

Here

$$
\begin{aligned}
\nabla_{\rho} w\left(p_{T}, p_{Y}, z\right) & \equiv \nabla_{z_{\rho}} w\left(p_{T}, p_{Y}, z\right) \\
\nabla_{y} \theta\left(y\left(p_{Y}, z\right), z\right) & \left.\equiv \nabla_{y} \theta(y, z)\right|_{y=y\left(p_{Y}, z\right)} \\
\nabla_{\rho} \theta\left(y\left(p_{Y}, z\right), z\right) & \left.\equiv \nabla_{z_{\rho}} \theta(y, z)\right|_{y=y\left(p_{Y}, z\right)} .
\end{aligned}
$$

Consider two elements of $Z, Z_{i}$ and $Z_{j}$ and impose the following quantile invariance restrictions.

$$
\begin{array}{rlll}
\nabla_{i} Q_{S \mid Z}\left(p_{Y}, z\right) & = & 0 & \nabla_{j} Q_{S \mid Z}(p, z)=0 \\
\left.\nabla_{i} Q_{W \mid S Z}\left(p_{T}, s, z\right)\right|_{s=s\left(p_{Y}, z\right)} & = & 0 & \left.\nabla_{j} Q_{W \mid S Z}\left(p_{T}, s, z\right)\right|_{s=s\left(p_{Y}, z\right)}=0
\end{array}
$$

The first pair of restrictions implies that

$$
\nabla_{i} s\left(p_{Y}, z\right)=0 \quad \nabla_{j} s\left(p_{Y}, z\right)=0
$$

and the restrictions together imply that

$$
\nabla_{i} w\left(p_{T}, p_{Y}, z\right)=0 \quad \nabla_{j} w\left(p_{T}, p_{Y}, z\right)=0 .
$$

Now impose the "local order condition":

$$
\left.\nabla_{i} \theta(y, z)\right|_{y=y\left(p_{Y}, z\right)}=0
$$

which requires the index to be insensitive to variations in $z_{i}$ when $Y$ is held constant at $Y=y\left(p_{Y}, z\right)$ and $Z=z$. The variable $Z_{i}$ is essentially an instrumental variable. The implication of the quantile invariance condition with respect to $Z_{i}$

$$
\nabla_{i} w\left(p_{T}, p_{Y}, z\right)=0
$$


and the order condition, imply that, on setting $\rho=i$ in (11), there is simplification of the expression for the $z_{i}$ derivative of $t\left(p_{T}, p_{Y}, z\right)$, as follows,

$$
\nabla_{i} t\left(p_{T}, p_{Y}, z\right)=\nabla_{\theta} J^{-1}\left(w\left(p_{T}, p_{Y}, z\right), \theta\left(y\left(p_{Y}, z\right), z\right)\right) \nabla_{y} \theta\left(y\left(p_{Y}, z\right), z\right) \nabla_{i} y\left(p_{Y}, z\right)
$$

from which, as long as the "local rank condition"

$$
\nabla_{i} y\left(p_{Y}, z\right) \neq 0
$$

holds:

$$
\frac{\nabla_{i} t\left(p_{T}, p_{Y}, z\right)}{\nabla_{i} y\left(p_{Y}, z\right)}=\nabla_{\theta} J^{-1}\left(w\left(p_{T}, p_{Y}, z\right), \theta\left(y\left(p_{Y}, z\right), z\right)\right) \nabla_{y} \theta\left(y\left(p_{Y}, z\right), z\right)
$$

Setting $\rho=j$ in (11) and using the following implication of the quantile invariance restrictions

$$
\nabla_{j} w\left(p_{T}, p_{Y}, z\right)=0
$$

results in:

$$
\begin{aligned}
\nabla_{j} t\left(p_{T}, p_{Y}, z\right)= & \nabla_{\theta} J^{-1}\left(w\left(p_{T}, p_{Y}, z\right), \theta\left(y\left(p_{Y}, z\right), z\right)\right) \nabla_{y} \theta\left(y\left(p_{Y}, z\right), z\right) \nabla_{j} y\left(p_{Y}, z\right) \\
& +\nabla_{\theta} J^{-1}\left(w\left(p_{T}, p_{Y}, z\right), \theta\left(y\left(p_{Y}, z\right), z\right)\right) \nabla_{j} \theta\left(y\left(p_{Y}, z\right), z\right)
\end{aligned}
$$

and so, on using (12),

$\nabla_{j} t\left(p_{T}, p_{Y}, z\right)-\frac{\nabla_{i} t\left(p_{T}, p_{Y}, z\right)}{\nabla_{i} y\left(p_{Y}, z\right)} \nabla_{j} y\left(p_{Y}, z\right)=\nabla_{\theta} J^{-1}\left(w\left(p_{T}, p_{Y}, z\right), \theta\left(y\left(p_{Y}, z\right), z\right)\right) \nabla_{j} \theta\left(y\left(p_{Y}, z\right), z\right)$

and, on combining (12) and (13),

$$
\frac{\nabla_{i} t\left(p_{T}, p_{Y}, z\right)}{\nabla_{j} t\left(p_{T}, p_{Y}, z\right) \nabla_{i} y\left(p_{Y}, z\right)-\nabla_{i} t\left(p_{T}, p_{Y}, z\right) \nabla_{j} y\left(p_{Y}, z\right)}=\frac{\left.\nabla_{y} \theta(y, z)\right|_{y=y\left(p_{Y}, z\right)}}{\left.\nabla_{j} \theta(y, z)\right|_{y=y\left(p_{Y}, z\right)}}
$$

which is the ratio of derivatives of the index whose identification is sought, as set out in equation (8).

The value of $y\left(p_{Y}, z\right)$ has already been shown to be identifiable - see equation (9). If $Z_{i}$ and $Z_{j}$ show continuous variation at the point $Z=z$ and if the relevant quantile invariance conditions hold in a neighbourhood of a point $z$, then the value of $y\left(p_{Y}, z\right)$ in a neighbourhood of $z$ is identified. From this it follows that at $z$ the derivatives $\nabla_{i} y\left(p_{Y}, z\right)$ and $\nabla_{j} y\left(p_{Y}, z\right)$ are identified, that is, in admissible structures:

$$
\begin{aligned}
& \nabla_{i} y\left(p_{Y}, z\right)=y_{\nabla_{i}}^{*} \Rightarrow \nabla_{i} Q_{Y \mid Z}\left(p_{Y}, z\right)=y_{\nabla_{i}}^{*} \\
& \nabla_{j} y\left(p_{Y}, z\right)=y_{\nabla_{j}}^{*} \Rightarrow \nabla_{j} Q_{Y \mid Z}\left(p_{Y}, z\right)=y_{\nabla_{j}}^{*}
\end{aligned}
$$

The ratio of derivatives in (10) is therefore identified if the $z_{i}$ and $z_{j}$ derivatives of the function $t\left(p_{T}, p_{Y}, z\right)$ are identified. It is now shown that this is the case.

4.3. Identification of $t\left(p_{T}, p_{Y}, z\right)$ and its derivatives. It is first shown that under the conditions stated the value of $t\left(p_{T}, p_{Y}, z\right)$ is identified, in particular, that in admissible structures:

$$
t\left(p_{T}, p_{Y}, z\right)=t^{*} \Rightarrow Q_{T \mid Y Z}\left(p_{T}, Q_{Y \mid Z}\left(p_{Y}, z\right), z\right)=t^{*} .
$$

First note that

$$
t\left(p_{T}, p_{Y}, z\right)=J^{-1}\left(w\left(p_{T}, p_{Y}, z\right), \theta\left(h\left(z, s\left(p_{Y}, z\right)\right), z\right)\right)
$$


where, recall,

$$
w\left(p_{T}, p_{Y}, z\right)=Q_{W \mid S Z}\left(p_{T}, s\left(p_{Y}, z\right), z\right) .
$$

The equivariance property of quantiles implies that

$$
t\left(p_{T}, p_{Y}, z\right)=Q_{T \mid S Z}\left(p_{T}, s\left(p_{Y}, z\right), z\right) .
$$

Since $h(z, s)$ is strictly increasing in $s$ at $z$, the two events

$$
\left\{S=s\left(p_{Y}, z\right) \cap Z=z\right\} \quad\left\{Y=y\left(p_{Y}, z\right) \cap Z=z\right\}
$$

are identical, and therefore,

$$
t\left(p_{T}, p_{Y}, z\right)=Q_{T \mid Y Z}\left(p_{T}, y\left(p_{Y}, z\right), z\right) .
$$

It has already been shown that the value of $y\left(p_{Y}, z\right)$ is identifiable - see equation (9) - and it therefore follows that, in admissible structures,

$$
t\left(p_{T}, p_{Y}, z\right)=t^{*} \Rightarrow Q_{T \mid Y Z}\left(p_{T}, Q_{Y \mid Z}\left(p_{Y}, z\right), z\right)=t^{*}
$$

and so the value of $t\left(p_{T}, p_{Y}, z\right)$ is identified.

If $Z_{i}$ and $Z_{j}$ show continuous variation at the point $Z=z$ and if the relevant quantile invariance conditions hold in a neighbourhood of a point $z$, then the value of $t\left(p_{T}, p_{Y}, z\right)$ in a neighbourhood of $z$ is identified. From this it follows that at $z$ the derivatives $\nabla_{i} t\left(p_{T}, p_{Y}, z\right)$ and $\nabla_{j} t\left(p_{T}, p_{Y}, z\right)$ are identified, that is, in admissible structures:

$$
\begin{aligned}
& \nabla_{i} t\left(p_{T}, p_{Y}, z\right)=t_{\nabla_{i}}^{*} \Rightarrow \nabla_{i} Q_{T \mid Y Z}\left(p_{T}, Q_{Y \mid Z}\left(p_{Y}, z\right), z\right)=t_{\nabla_{i}}^{*} \\
& \nabla_{j} t\left(p_{T}, p_{Y}, z\right)=t_{\nabla_{j}}^{*} \Rightarrow \nabla_{j} Q_{T \mid Y Z}\left(p_{T}, Q_{Y \mid Z}\left(p_{Y}, z\right), z\right)=t_{\nabla j}^{*}
\end{aligned}
$$

This, together with the results (14) and (15), implies that in admissible structures:

$$
\begin{gathered}
\left.R_{Y j}(y, z)\right|_{y=y\left(p_{Y}, z\right)}=R^{*} \\
\Rightarrow \\
\frac{\nabla_{i} Q_{T \mid Y Z}\left(p_{T}, Q_{Y \mid Z}\left(p_{Y}, z\right), z\right)}{\nabla_{j} Q_{T \mid Y Z}\left(p_{T}, Q_{Y \mid Z}\left(p_{Y}, z\right), z\right) \nabla_{i} Q_{Y \mid Z}\left(p_{Y}, z\right)-\nabla_{i} Q_{T \mid Y Z}\left(p_{T}, Q_{Y \mid Z}\left(p_{Y}, z\right), z\right) \nabla_{j} Q_{Y \mid Z}\left(p_{Y}, z\right)}=R^{*}
\end{gathered}
$$

which demonstrates the identification of the value of $\left.R_{Y j}(y, z)\right|_{y=y\left(p_{Y}, z\right)}$.

4.4. Implications for estimation. There are two potential sources of $Z$-driven variation in the iterated conditional quantile functions that appear in the identifying equation above, and so, for example,

$$
\begin{gathered}
\nabla_{i} Q_{T \mid Y Z}\left(p_{T}, Q_{Y \mid Z}\left(p_{Y}, z\right), z\right)=\left.\nabla_{y} Q_{T \mid Y Z}\left(p_{T}, y, z\right)\right|_{y=Q_{Y \mid Z}\left(p_{Y}, z\right)} \nabla_{i} Q_{Y \mid Z}\left(p_{Y}, z\right) \\
+\left.\nabla_{i} Q_{T \mid Y Z}\left(p_{T}, y, z\right)\right|_{y=Q_{Y \mid Z}\left(p_{Y}, z\right)}
\end{gathered}
$$

Estimation of the value of the ratio of index function derivatives is straightforwardly achieved by first estimating the iterated conditional quantile functions $Q_{T \mid Y Z}$ and $Q_{Y \mid Z}$ and their derivatives, then plugging these estimates into (17) using equation (18). Quantile regression estimation technology is in a quite advanced state so it would be superfluous to go into the fine detail of the estimation procedure to which the identification result of this paper points. However, there are three issues worth considering.

First, if the quantile invariance, local order and local rank conditions hold for more than one covariate $Z_{i}$ then (17) is true for more than one choice of $Z_{i}$ and the value of $\left.R_{Y j}(y, z)\right|_{y=y\left(p_{Y}, z\right)}$ is over identified. Efficiency considerations may lead one to combine 
alternative estimates got using just identifying subsets of restrictions, for example using a minimum distance procedure. Alternatively this opens up the possibility of testing a just identifying subset of the restrictions of the model.

Second, if the quantile invariance, local order and local rank conditions hold for two or more values of $\left(z, p_{Y}, p_{T}\right)$ which yield the same value of $\left.R_{Y j}(y, z)\right|_{y=y\left(p_{Y}, z\right)}$ then the value of $\left.R_{Y j}(y, z)\right|_{y=y\left(p_{Y}, z\right)}$ is overidentified and again there are possibilities for improved efficiency in estimation and for testing the specification of the model. This can happen if elements of $z$ to which the index is insensitive can be adjusted to compensate for variations in $p_{Y}$, leaving $y\left(p_{Y}, z\right)$ unchanged.

Third, even though an index function derivative ratio is identified by the model considered in this paper, this ratio may not be easy to estimate accurately in even moderate sized samples if the semi-parametric specification of this paper is maintained. Typically, rates of convergence of estimated derivatives of quantile regression functions are slow in the absence of parametric restrictions. This might lead one to consider estimating an $a v$ erage index function derivative ratio with averaging taking place over some set of values of the covariates, that is estimating structural features of the sort

$$
\left.\int R_{Y j}(y, z)\right|_{y=y\left(p_{Y}, z\right)} d W(z)
$$

for some function $W(z)$. Of course the quantile invariance, order (exclusion) and rank (inclusion) restrictions would have to be maintained across all values of $z$ given nonzero weight by $W(z)$. Whether such averages are of interest in practice is a matter for case-by-case consideration. An alternative is to maintain fairly strong, but somewhat flexible additional parametric restrictions and to regard the results as contingent on the correctness of these restrictions, which, note, since they are overidentifying, are in principle testable.

So far identification has been considered when restrictions may be local to a particular value of $p_{T}$. However, on further examination, just local to $p_{T}$ restrictions on the conditional quantiles of $W$ given $X$ appear rather difficult to maintain in practice, an issue explored in the next Section.

4.5. Local to $p_{T}$ restrictions on conditional quantiles. In the development of the identifying model $^{8}$ in Section 3 conditional $p$-quantiles of $T$ were considered. Under the monotonicity restrictions embodied in the model these were equal to the value delivered by the structural function for $T$ evaluated at $W(V, U)=w(p, x)$ where

$$
w(p, x) \equiv Q_{W \mid X}(p, x)
$$

is the conditional $p$-quantile of $W(V, U)$ given $X=x$ and

$$
W(V, U) \equiv-U \log (1-V)
$$

The identifying restrictions of the model include quantile invariance restrictions of the sort

$$
\nabla_{i} Q_{W \mid X}(p, x)=0
$$

where $\nabla_{i}$ indicates partial differentiation with respect to $x_{i}$.

There would be considerable flexibility if such restrictions could be maintained at one value of $p$ but not at others. Unfortunately such a local restriction at one value of $p$ on $\nabla_{i} Q_{W \mid X}(p, x)$ implies a "global" restriction on the distribution function $F_{U \mid X}$. It is difficult to imagine situations in which this global restriction could be maintained at one value of $p$ and not at all other values of $p$.

\footnotetext{
${ }^{8}$ The discussion of this Section also applies to the development of Section 4 with appropriate changes in notation.
} 
It is shown in Annex 3 that the conditional quantile function of $W$ given $X$ is

$$
Q_{W \mid X}(p, x)=L^{-1}(1-p, x)
$$

where $L^{-1}$ is the inverse function of the function $L(w, x)$ with respect to $w$, and $L(w, x)$ is the Laplace transform of the distribution of $U^{-1}$ given $X$, that is

$$
L(w, x)=\int_{0}^{\infty} \exp (-w / u) d F_{U \mid X}(u \mid x) .
$$

It is further shown in Annex 3 that

$$
\nabla_{i} Q_{W \mid X}(p, x)=\frac{\left.\nabla_{i} L(w, x)\right|_{w=Q_{W \mid X}(p, x)}}{f_{W \mid X}\left(Q_{W \mid X}(p, x) \mid x\right)}
$$

where $f_{W \mid X}$ is the probability density function of $W$ given $X$, and

$$
\left.\nabla_{i} L(w, x)\right|_{w=Q_{W \mid X}(p, x)}=\int_{0}^{\infty} \exp \left(-Q_{W \mid X}(p, x) / u\right) d\left(\nabla_{i} F_{U \mid X}(u \mid x)\right) .
$$

The quantile invariance condition $\nabla_{i} Q_{W \mid X}(p, x)=0$ clearly holds at $x$ for all $p$ if $\nabla_{i} F_{U \mid X}(u \mid x)=0$ for all $u$, which is in the nature of a local independence condition. It is difficult to imagine cases in which, for some $u, \nabla_{i} F_{U \mid X}(u \mid x) \neq 0$ and yet, for some $p$, $\left.\nabla_{i} L(w, x)\right|_{w=Q_{W \mid X}(p, x)}=0$.

In summary: local to $p$ quantile invariance restrictions on $Q_{W \mid X}(p, x)$ are difficult to maintain, and a quantile invariance restriction on $Q_{W \mid X}(p, x)$ for variations in $X_{i}$ can only reasonably be maintained if the restriction that $U$ is independent of $X_{i}$ is maintained, at least for variations in $X$ in a neighbourhood of the value, $x$, of interest.

4.6. Censoring. So far no mention has been made of censoring which is a common feature of data generated by duration processes. In the context of the Hurwicz structural form representation, censoring is captured by writing the structural equation system as

$$
\begin{aligned}
T & =C\left(J^{-1}(W, \theta(Y, Z))\right) \\
Y & =h(Z, S)
\end{aligned}
$$

where $C(\cdot)$ is a weakly increasing function capturing the type of censoring to be considered. For example if there is right censoring at some value $c$ then

$$
\begin{aligned}
& C(t)=t, \quad t \leq c \\
& C(t)=c, \quad t>c .
\end{aligned}
$$

Consider the analysis of identification under exogeneity. In that analysis, with censoring, the function $t(p, x)$ will be defined as

$$
t(p, x)=C\left(J^{-1}(w(p, x), \theta(x))\right)
$$

and the result

$$
t(p, x)=Q_{T \mid X}(p, x)
$$

still holds as long as we use the following definition of a conditional $p$-quantile for a random variable, $A$ say, given $X$ with possibly non-differentiable distribution function $F_{A \mid X}$.

$$
Q_{A \mid X}(p, x)=\inf \left\{a: F_{A \mid X}(a, x) \geq p\right\}
$$


The derivatives of $t(p, x)$ with respect to $x_{i}$ and $x_{j}$ under the local exogeneity restrictions will be as follows.

$$
\begin{aligned}
t_{i}(p, x) & =\nabla_{t} C\left(J^{-1}(w(p, x), \theta(x))\right) \nabla_{\theta} J^{-1}(w(p, x), \theta(x)) \nabla_{i} \theta(x) \\
t_{j}(p, x) & =\nabla_{t} C\left(J^{-1}(w(p, x), \theta(x))\right) \nabla_{\theta} J^{-1}(w(p, x), \theta(x)) \nabla_{j} \theta(x)
\end{aligned}
$$

In the analysis of identification under exogeneity the ratio of index function derivatives is obtained as

$$
\frac{\nabla_{i} \theta(x)}{\nabla_{j} \theta(x)}=\frac{t_{i}(p, x)}{t_{j}(p, x)}
$$

a result which applies as long as the derivative of the censoring function is non-zero at the point at which identification is sought. So, for the identification result to hold, $p$ and $x$ must be chosen so that $J^{-1}(w(p, x), \theta(x))$ falls on a differentiable segment of the censoring function, that is at point at which censoring does not induce discreteness in the distribution of the observed duration.

Similar considerations apply in the analysis of identification under endogeneity, and there is an additional consideration. The analysis of identification under endogeneity rests on the restriction requiring the function $h(Z, S)$ to be strictly increasing in $S$ at the value of $Z$ considered. In practice ${ }^{9}$ this requires $Y$ to be continuously distributed at the value of $(p, z)$ considered, and censoring of $Y$, if it occurs, must not remove this property.

In summary, the identification result under endogeneity may apply when $T$ and (or) $Y$ are censored, but censoring must not result in either $T$ or $Y$ being non-continuously distributed at the value of $\left(p_{T}, p_{Y}, z\right)$ considered.

\section{CONCLUDING REMARKS}

The relative sensitivity of a single index in a duration model to variations in its possibly endogenous determinants has been shown to be identifiable under weak semi-parametric conditions. The identification result is constructive in the sense that it points to an analogue estimator of ratios of derivatives of an index function an estimator which is a functional of estimated iterated conditional quantile regression functions.

If the endogenous variable appearing in the hazard function is interpreted as the duration spent in a state at some earlier time then the results of the paper are relevant when considering identification in semi-parametrically specified panel duration models.

To see this, consider a panel of length two and suppose $T_{1}$ and $T_{2}$ are two durations modelled as in this paper with integrated hazard functions respectively $J_{1}\left(t_{1}, \theta_{1}(z)\right) / u_{1}$ and $J_{2}\left(t_{2}, \theta_{2}\left(t_{1}, z\right)\right) / u_{2}$ where $u_{1}$ and $u_{2}$ are values of latent unobservable random variables. Then in Hurwicz structural form we have

$$
\begin{aligned}
& T_{2}=J_{2}^{-1}\left(W_{2}, \theta_{2}\left(T_{1}, Z\right)\right) \\
& T_{1}=J_{1}^{-1}\left(W_{1}, \theta_{1}(Z)\right)
\end{aligned}
$$

where

$$
\begin{aligned}
& W_{2}=-U_{2} \log \left(1-V_{2}\right) \\
& W_{2}=-U_{1} \log \left(1-V_{1}\right) .
\end{aligned}
$$

\footnotetext{
${ }^{9}$ With $h(Z, S)$ strictly increasing in $S$, the distribution of $Y$ is continuous unless $S$ has a discrete distribution. But when $h(Z, S)$ is strictly increasing in $S$ and $S$ has a discrete distribution, varaiations in $Z$ have no impact on the probability masses on the points of support of the distribution of $Y$, their only effect being to change the location of the points of support. For practical purposes the analysis of this paper requires the endogenous $Y$ to be continuously distributed.
} 
Here $V_{1}$ and $V_{2}$ are independently uniformly distributed each on $(0,1)$, by construction distributed independently of $\left(U_{1}, U_{2}, Z\right)$, and $U_{1}$ and $U_{2}$ are jointly distributed random variables. This is in the class of triangular nonseparable simultaneous equations structures studied in this paper and so the identification conditions developed in this paper apply. Extension to longer panels is straightforward.

\section{REFERENCES}

Chaudhuri, P., K. Doksum and A. Samarov (1997): "On average derivative quantile regression," Annals of Statistics, 25, 715-744.

Chesher, A.D., And T. Lancaster (1984) "Simultaneous Equations with Endogenous Hazards," in Studies in Labor Market Dynamics, edited by G.B. Neumann and N.C. Westergard-Nielsen, Springer Verlag.

Chesher, A.D., (2001a): "Exogenous impact and conditional quantile functions," Centre for Microdata Methods and Practice Working Paper 01/01.

Chesher, A.D., (2001b): "Quantile driven identification of structural derivatives," Centre for Microdata Methods and Practice Working Paper 08/01.

Chesher, A.D., (2002a): "Local identification in nonseparable models," Centre for Microdata Methods and Practice Working Paper 05/02.

Chesher, A.D., (2002b): "Instrumental values," Centre for Microdata Methods and Practice Working Paper 17/02.

HAN, A., (1987): "A non-parametric analysis of transformations," Journal of Econometrics, 35, 191-209.

HuRwicz, L. (1950): "Generalization of the concept of identification," in Statistical inference in dynamic economic models. Cowles Commission Monograph 10, New York, John Wiley.

KAHN, S., (2001): "Two-stage rank estimation of quantile index models," Journal of Econometrics, 100, 319-355.

Koenker, R., And G. Bassett JR. (1978): "Regression quantiles," Econometrica, 46, 33-50.

Koenker, R., And O. Geling (2001): "Reappraising medfly longevity: a quantile regression survival analysis," Journal of the American Statistical Association, 96, 458468.

Lancaster, T., And A.D. Chesher (1983) "Econometrics of reservation wages," Econometrica, 51, 1661-1676.

LANCASTER, T., (1985): "Simultaneous equations models in applied search theory," Journal of Econometrics, 28(1), 113-126.

Manski, C., (1988): "Identification of binary response models," Journal of the American Statistical Association, 83, 729-738.

Matzkin, R.L., (1999): "Nonparametric estimation of nonadditive random functions," Invited lecture on New Development in the Estimation of Preferences and Production Functions, 1999 Latin American Meeting of the Econometric Society.

Newey, W.K., AND T. Stoker, (1993): "Efficiency of weighted average derivative estimators and index models," Econometrica, 61, 1199-1223.

Powell, J., Stock, J., And T. Stoker, (1989): "Semiparametric estimation of index coefficients," Econometrica, 57, 1403-1430.

Roehrig, C.S., (1988): "Conditions for identification in nonparametric and parametric models", Econometrica 56, 433-447. 


\section{ANNEX 1}

\section{A. The restrictions of the model for identification of $R_{i j}(x)$ with local exogeneity.}

1. Hazard function. The hazard function for scalar, absolutely continuously distributed positive valued $T$ given $k$ element $X=x$ and scalar positive valued $U=u$ is

$$
\lambda_{T \mid X U}(t \mid x, u)=j(t, \theta(x)) / u
$$

where $\theta(x)$ is a scalar function of $x$, and $j(t, \theta(x))>0$ for all $t$ and $x$.

2. Continuous variation. Consider two variables $x_{i}$ and $x_{j}$. These variables admit continuous variation in a neighbourhood of the value $x$ of interest.

3. Differentiability. At a value $x$ of interest the hazard function is a differentiable function of $t$ and of $\theta(x)$. At a value $x$ of interest, the index function, $\theta(x)$ is a differentiable function of $x_{i}$ and $x_{j}$. Define the random variable:

$$
W=-U \log (1-V)
$$

where $V$ is uniformly distributed on $(0,1)$ independently of $(U, X)$. Let $Q_{W \mid X}(p, x)$ be the conditional $p$-quantile of $W$ given $X=x$. At a value of $p$ and $x$ of interest, $Q_{W \mid X}(p, x)$ is a differentiable function of $x_{i}$ and $x_{j}$.

4. Nonzero derivatives. At a value $x$ of interest the derivative $\nabla_{j} \theta(x) \neq 0$ and $\nabla_{\theta} j(t, \theta(x)) \neq 0$.

5. Local quantile invariance. For $x^{*}$ in a neighbourhood of the value $x$ of interest and for some value of $p$

$$
\nabla_{i} Q_{W \mid X}\left(p, x^{*}\right)=0 \quad \nabla_{j} Q_{W \mid X}\left(p, x^{*}\right)=0
$$

where $W \equiv-U \log (1-V)$ and $V$ is uniformly distributed on $(0,1)$ and $U$ given $X=x$ is, by construction, distributed independently of $V$.

\section{ANNEX 2}

\section{B. The restrictions of the model for identification of $R_{Y j}(y, z)$ with local} endogeneity

1. Hazard function. The hazard function for scalar, absolutely continuously distributed positive valued $T$ given $k$ element $Z=z$, scalar $Y=y$, and scalar positive valued $U=u$ is

$$
\lambda_{T \mid Y Z U}(t \mid y, z, u)=j(t, \theta(y, z)) / u
$$

where $\theta(y, z)$ is a scalar function of $y$ and $z$, and $j(t, \theta(y, z))>0$ for all $t, y$ and $z$.

2. Endogenous variate. The endogenous variate $Y$ is generated by the equation

$$
Y=h(Z, S)
$$

where $h(Z, S)$ is strictly monotonic (normalised increasing) in $S$ and $S$ is a random variable. 
3. Continuous variation. Restrictions are placed on the behaviour of functions as values of $Y$ and of two elements of $Z, Z_{i}$ and $Z_{j}$ alter. The variates $Y, Z_{i}$ and $Z_{j}$ admit continuous variation in a neighbourhood of a value $(y, z)$ of interest.

4. Differentiability. The hazard function is a differentiable function of $t$ and of $\theta(y, z)$. At a value $(y, z)$ of interest, the index function, $\theta(y, z)$ is a differentiable function of $y, z_{i}$ and $z_{j}$. Let $Q_{S \mid Z}\left(p_{Y}, z\right)$ be the conditional $p_{Y}$-quantile of $S$ given $Z=z$. At values $p_{Y}$ and $z$ of interest $Q_{S \mid Z}\left(p_{Y}, z\right)$ is a differentiable function of $z_{i}$ and $z_{j}$. For a chosen value of $p_{Y}$ and $z$ define $s\left(p_{Y}, z\right)=Q_{S \mid Z}\left(p_{Y}, z\right)$. Define the random variable:

$$
W=-U \log (1-V)
$$

where $V$ is uniformly distributed on $(0,1)$ independently of $(U, X)$. Let $Q_{W \mid S Z}\left(p_{T}, s, z\right)$ be the conditional $p_{T}$-quantile of $W$ given $S=s$ and $Z=x$. At values of $p_{T}$ and $z$ of interest and at $s=s\left(p_{Y}, z\right), Q_{W \mid S Z}\left(p_{T}, s, z\right)$ is a differentiable function of $s, z_{i}$ and $z_{j}$.

5. Nonzero derivatives. At the value $(y, z)$ of interest $\nabla_{j} \theta(y, z) \neq 0$ and $\nabla_{\theta} j(t, \theta(x)) \neq$ 0 .

6. Local quantile invariance. For $z^{*}$ in a neighbourhood of the value $z$ of interest and for a value $p_{T}$ of interest,

$$
\begin{array}{rlll}
\nabla_{i} Q_{S \mid Z}\left(p_{Y}, z^{*}\right) & = & 0 & \nabla_{j} Q_{S \mid Z}\left(p_{Y}, z^{*}\right)=0 \\
\left.\nabla_{i} Q_{W \mid S Z}\left(p_{T}, s, z^{*}\right)\right|_{s=s\left(p_{Y}, z\right)} & = & 0 & \left.\nabla_{j} Q_{W \mid S Z}\left(p_{T}, s, z^{*}\right)\right|_{s=s\left(p_{Y}, z\right)}=0 .
\end{array}
$$

7. Local order condition. Define $y\left(p_{Y}, z\right)=h\left(z, s\left(p_{Y}, z\right)\right)$. At $y=y\left(p_{Y}, z\right)$ and the chosen value of $z$

$$
\left.\nabla_{i} \theta(y, z)\right|_{y=y\left(p_{Y}, z\right)}=0 .
$$

8. Local rank condition. At the value of $z$ of interest

$$
\nabla_{i} y\left(p_{Y}, z\right) \neq 0 .
$$

\section{ANNEX 3}

The relationship between the distributions of $W$ and $V$.

The random variable $W$, which is the sole (combined) source of stochastic variation in the structural equation for the duration $T$ under the proportionate heterogeneity restriction, is defined as

$$
W(V, U) \equiv-U \log (1-V)
$$

where $V$ is uniformly distributed on $(0,1)$ and $U$ is the source of heterogeneity which has distribution function $F_{U \mid X}$ and is by construction distributed independently of $V$. This Annex develops the relationship between the distributions of $W$ and $U$.

\section{The distribution function of $W$ given $X$}

First note that

$$
\begin{aligned}
P[W \leq w \mid X=x] & =P[-U \log (1-V) \leq w \mid X=x] \\
& =P\left[V \leq 1-\exp \left(-\frac{w}{U}\right) \mid X=x\right] \\
& =E_{U \mid X=x}\left[P\left[V \leq 1-\exp \left(-\frac{w}{U}\right) \mid U, X=x\right]\right]
\end{aligned}
$$


and since $V$ is uniformly distributed on $(0,1)$,

$$
P[W \leq w \mid X=x]=E_{U \mid X=x}\left[1-\exp \left(-\frac{w}{U}\right)\right]
$$

that is, the conditional distribution function of $W$ given $X$ is

$$
F_{W \mid X}(w \mid x)=1-L(w, x)
$$

where $L(w, x)$ is the Laplace transform of the distribution of $\tilde{U}$ given $X=x$ and $\tilde{U} \equiv U^{-1}$, that is:

$$
L(w, x)=\int_{0}^{\infty} \exp (-w / u) d F_{U \mid X}(u \mid x) .
$$

\section{The conditional quantile function of $W$ given $X$}

The conditional quantile function of $W$ given $X$ is therefore

$$
Q_{W \mid X}(p, x)=L^{-1}(1-p, x)
$$

where $L^{-1}$ is the inverse function of the function $L$, that is $L^{-1}(z, x)$ is the solution to

$$
z=L\left(L^{-1}(z, x), x\right) .
$$

\section{Derivatives of the conditional quantile function}

The derivative of $Q_{W \mid X}(p, x)$ with respect to $x_{i}$ is therefore

$$
\nabla_{i} Q_{W \mid X}(p, x)=\nabla_{i} L^{-1}(1-p, x) .
$$

We have, setting $z=1-p$ in (A3.2),

$$
0=\left.\nabla_{w} L(w, x)\right|_{w=Q_{W \mid X}(p, x)} \nabla_{i} Q_{W \mid X}(p, x)+\left.\nabla_{i} L(w, x)\right|_{w=Q_{W \mid X}(p, x)}
$$

and so, on noting that, from (A3.1)

$$
\left.\nabla_{w} L(w, x)\right|_{w=Q_{W \mid X}(p, x)}=-f_{W \mid X}\left(Q_{W \mid X}(p, x) \mid x\right)
$$

where $f_{W \mid X}$ is the conditional probability density function of $W$ given $X$. Finally, there is the following expression for the derivative with respect to $x_{i}$ of the conditional $p$-quantile function of $W$ given $X$ :

$$
\nabla_{i} Q_{W \mid X}(p, x)=\frac{\left.\nabla_{i} L(w, x)\right|_{w=Q_{W \mid X}(p, x)}}{f_{W \mid X}\left(Q_{W \mid X}(p, x) \mid x\right)}
$$

where

$$
\left.\nabla_{i} L(w, x)\right|_{w=Q_{W \mid X}(p, x)}=\int_{0}^{\infty} \exp \left(-Q_{W \mid X}(p, x) / u\right) d\left(\nabla_{i} F_{U \mid X}(u \mid x)\right) .
$$

Clearly, if, for some $x, \nabla_{i} F_{U \mid X}(u \mid x)=0$ for all $u$, which is in the nature of a local independence condition, then $\nabla_{i} Q_{W \mid X}(p, x)=0$ for all $p$. 